\title{
Stellar and circumstellar evolution of long period variable stars
}

\author{
M. O. Mennessier ${ }^{1}$ and X. Luri ${ }^{2}$ \\ 1 Université de Montpellier II and CNRS, G.R.A.A.L., cc072, 34095 Montpellier Cedex 5, France \\ 2 Departament d'Astronomia i Meteorologia, Universitat de Barcelona, Avda. Diagonal 647, 08028 Barcelona, \\ Spain
}

Received 4 May 2001 / Accepted 26 September 2001

\begin{abstract}
In a first paper, HIPPARCOS astrometric and kinematic data were used to calibrate both infrared $K$ and IRAS luminosities at the same time as kinematic parameters of Long Period Variable stars (LPVs). Individual estimated absolute magnitudes and a probabilistic assignation to galactic populations were deduced from these calibrations for each LPV of our sample. Here we propose a scenario of simultaneous stellar and circumstellar evolution according to the galactic populations. The transitory states of S and Tc stars allow us to confirm the location of the first dredge-up at $M_{\mathrm{bol}}=-3.5$. There is also evidence suggesting that a previous enrichment in s-elements from a more evolved companion may accelerate the evolution along the AGB. The possible evolution to OH LPVs is included in this scenario, and any of these stars may have a mass at the limit of the capability for a $\mathrm{C}$ enrichment up to $\mathrm{C} / \mathrm{O}>1$. A list of bright massive LPVs with peculiar envelope and luminosity properties is proposed as Hot Bottom Burning candidates. The He-shell flash star, R Cen, is found to be exceptionally bright and could become, before leaving the AGB, a C-rich LPV brighter than the usual luminosity limit of carbon stars.
\end{abstract}

Key words. stars: variable: general - stars: evolution - stars: AGB and post-AGB

\section{Introduction}

LPVs are particularly interesting red giants for two main reasons. On the one hand, the brightest LPVs are luminous enough to be observed at large distances, providing information on the host galaxy (Van Loon et al. 1999a). On the other hand, although their precise ranges of masses and ages remain controversial, it is clear that they are large enough. Thus LPVs are very good tracers of the galactic history. Moreover the final evolution along the AGB, and peculiarly the carbon surface enrichment and the change of the envelope chemistry, is very complex. It depends on many factors (as convection, overshooting, internal chemical process, mass-loss, pulsation, etc.), the relative effects of which depend on the mass and metallicity, among other things.

In a previous paper (Mennessier et al. 2000), hereafter Paper I, HIPPARCOS astrometric data and multiwavelength photometric measurements of a sample of 800 LPVs (semi-regular a and b, irregular L and Mira with $\mathrm{O}, \mathrm{S}$ and $\mathrm{C}$ spectral types) were analyzed using the LM algorithm (Luri et al. 1996). $V, K$ and IRAS 12 and 25 luminosities were calibrated. The LM algorithm classified the stars according to the galactic population (associated with the initial mass and metallicity

Send offprint requests to: M. O. Mennessier, e-mail: Marie-Odile.Mennessier@graal .univ-montp2.fr of the stars) and to the circumstellar envelope thickness and expansion. Several groups were obtained in this classification:

- Bright disk (BD) and disk (disk 1 or D1) LPVs with bright and expanding envelopes;

- Not so young and massive disk population (disk 2 or D2) divided into two subgroups: one with a thin envelope (denoted $\mathrm{f}$ ) and another one with a bright and expanding envelope (denoted b);

- Old disk (OD) population, showing a similar separation ( $b$ and $\mathrm{f}$ ) according to envelope properties;

- Some LPVs were included in the extended disk (ED) population.

These groups were obtained by combining $\mathrm{K}$ and IRAS results.

From kinematic properties, the disk 1 population was found to be $1-4 \times 10^{9} \mathrm{yr}$ old, disk 2 population $4-8 \times 10^{9} \mathrm{yr}$ old and the old disk population older than $8 \times 10^{9} \mathrm{yr}$, up to $10^{10}$ yr or even more. An extended disk was assumed to be composed of very old, metal-deficient stars.

The lower limits of the main sequence initial mass, $\mathcal{M}_{\text {ms }}$, were estimated to be in the range $2-1.4 \mathcal{M}_{\odot}$, $1.4-1.15 \mathcal{M}_{\odot}$, and $1.15-1 \mathcal{M}_{\odot}$ for disk 1 , disk 2 and old disk populations respectively. Moreover, in Paper I, statistical estimates were done to quantify how much groups 
and various variability and spectral types attract or repel each other.

Each star of the sample was assigned to a galactic population and its individual $\mathrm{K}$ and IRAS 12 and 25 absolute magnitudes were estimated. A table with these values is available in electronic form at $\mathrm{CDS}^{1}$. They also are available in the ASTRID specialized database ${ }^{2}$.

In this paper, we use the estimated individual stellar absolute magnitudes $(K)$ together with properties of the circumstellar envelopes (deduced from IRAS absolute magnitudes) and the assigned galactic population to define an evolutive scenario of simultaneous stellar and circumstellar evolution of LPVs along the Asymptotic Giant Branch (AGB). We aimed to link the chemical evolution from O-rich to C-rich LPVs or $\mathrm{OH}$ emitters (through the intermediate states of $\mathrm{S}$ and/or Tc LPVs) and the stellar and circumstellar evolution, depending on the galactic population, i.e. on the initial mass, as discussed in Sect. 2.

In Sect. 3 we examine the first stages of O-rich LPVs and their correlation with initial mass. More precisely, we propose and critically study several possible explanations for the gap observed in the distribution of O-rich LPVs, separating those with and without a circumstellar shell.

Section 4 is dedicated to the brightest $\mathrm{O}$ and C-rich LPVs and points out candidates for peculiarities like Hot Bottom Burning (HBB). Special attention is given to the case of R Cen, a star in a He-shell flash.

Finally, a global stellar and circumstellar evolutive scenario is proposed in Sect. 5, which takes into account the differences between galactic populations and explains both chemical and variability-type changes.

\section{Chemical evolution}

It is well known that $K$ and IRAS absolute magnitudes reflect the properties of various parts of the star. The $K$ magnitude depends mainly on the characteristics of the stellar surface, whereas IRAS fluxes are linked to envelope thickness and dust composition. Using both types of magnitudes, information about the stellar and circumstellar properties can be obtained.

Here we mainly use the individual absolute magnitudes and assigned galactic populations estimated in Paper I. It is convenient to recall that our sample was found to be representative of the $L P V$ population as far as the kinematics and the brightest luminosities are concerned, but is under-representative for $K$ and IRAS faint stars (see Paper I).

\subsection{From O-rich to C-rich $L P V s$}

Figure 1 shows the distribution of the individual estimated $\mathrm{K}$ absolute magnitudes and $25-12^{3}$ indices deduced from

\footnotetext{
1 Via anonymous ftp to cdsarc.u-strasbg.fr (130.79.128.5) or via http://cdsweb.u-strasbg.fr/cgi-bin/qcat?J/A+A/380/198

2 http://astrid.graal.univ-montp2.fr

${ }^{3} m_{25}=2.07-2.5 \times \log F_{25}$ and $m_{12}=3.63-2.5 \times \log F_{12}$.
}

the estimated IRAS absolute magnitudes according to the assigned kinematical groups and spectral types. A bimodal distribution of stars with a deficit in the number of stars around $25-12=-0.2$ is clear, mainly for the lower mass stars (disk 2 and old disk). Section 3 will examine in detail this gap for O-rich stars. In the present section we focus on the fact that this area contains mainly C-rich stars belonging to disk population. Moreover it corresponds to the range of the (25-12) index with the lowest ratio of known variable stars among the IRAS sources with colors similar to LPVs colors (Paper I, Fig. 4).

This gap has already been reported by Habing (1989) and interpreted as the separation between stars with and without a circumstellar envelope. The deficit is very marked in regions I and II of Habing's IRAS color-color diagram $^{4}$, which correspond to non-variable and variable (mainly O-rich) stars, and slighter in region VII, which contains C-rich variable stars, in agreement with our results. Thus, stars with a thin circumstellar envelope (f) are clustered around 25-12=0 and almost all of them are O-rich LPVs. However, the kinematical study allowed us to assess the differences according to the initial mass of the stars.

The most massive stars can evolve to C-rich LPVs after a number of dredge-ups that enrich the external shells of the star in carbon. In these stars the $\mathrm{C} / \mathrm{O}$ ratio becomes larger than 1 and when it is around 1 , the star is an S star. At the same time, strong changes take place in the circumstellar envelope, which becomes dominated by C-rich grains. The 25-12 index increases in conformity with the loop in the IRAS (25-12, 60-25) color-color diagram predicted by Willems \& de Jong (1988) and calculated by Chan \& Kwok (1988). The areas occupied by O-rich and C-rich disk LPVs in Fig. 1 reflect this loop. Indeed the 25-12 index decreases from stars with a thin circumstellar envelope (f) to O-rich LPVs with a thick envelope (b) and increases again for C LPVs.

However, our luminosity calibrations suggest that the phenomenon does not strongly induce a difference in the $K$ distributions of O-rich and C-rich disk LPVs. Moreover, the ( $K$, IRAS) luminosity diagrams of Figs. 2 and 3 show that the loop is caused by both the decreasing 12 and 25 luminosities when the star becomes C-rich, but that this decrease is stronger in the 25 filter.

C-rich irregular and SRb stars mainly belong to the disk 1 population (Paper I, Sect. 6.4). They are mainly located at the upper end of the AGB. Therefore, the change from a more regular variable star (Mira) to a less regular one ( $\mathrm{L}$ or $\mathrm{SRb}$ ) may be associated with an increase in the non-linear behaviour of the massive pulsating LPVs due to interactions of the pulsation phenomenon and a very thick and dynamically unstable envelope.

\footnotetext{
4 [25]-[12] index is higher than ours by $1.56 \mathrm{mag}$.
} 

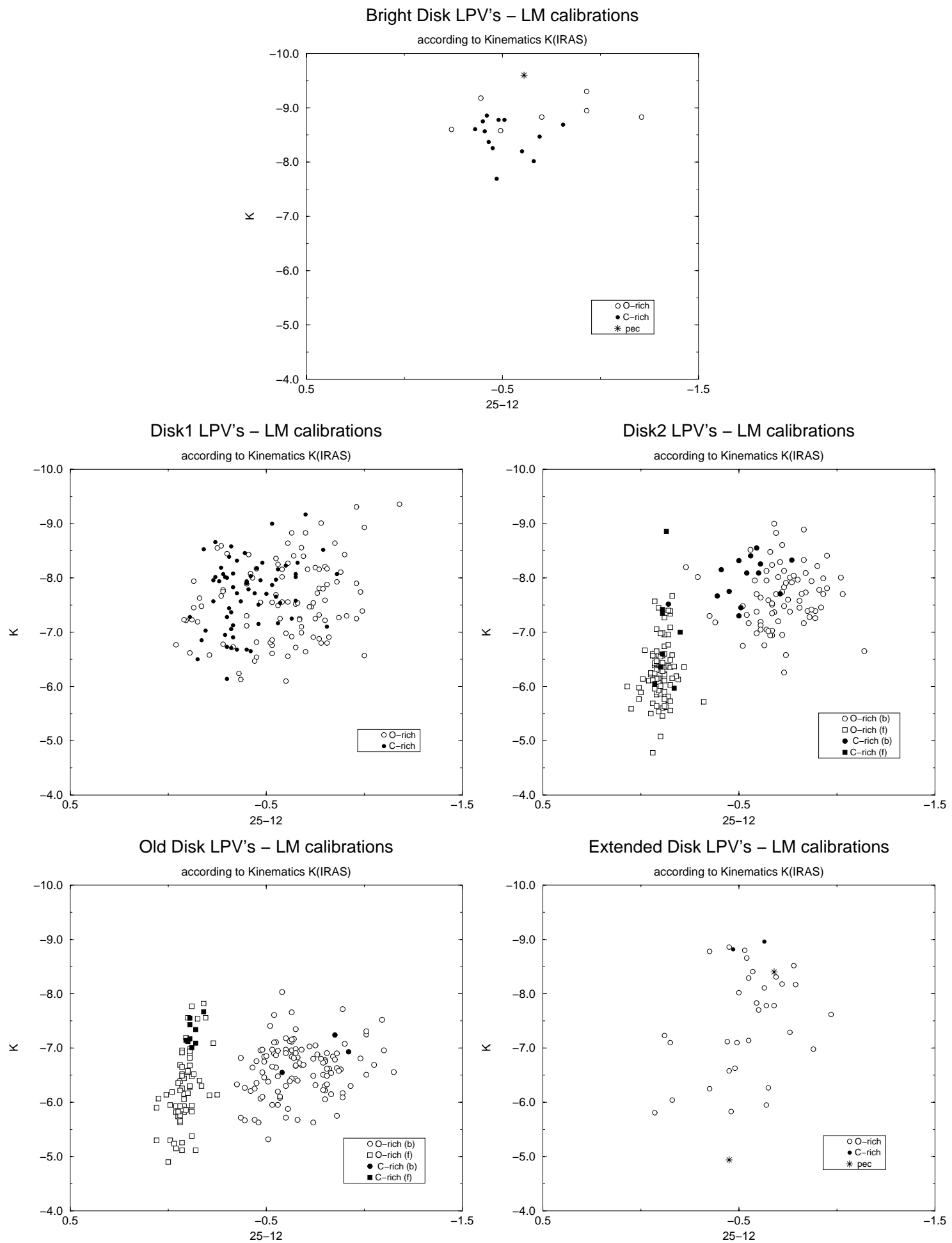

Fig. 1. Distribution of the individual estimated $K$ luminosities and 12-25 IRAS colors according to the assigned kinematical groups and to spectral types.

\subsection{Peculiar evolutive phases}

The above global evolutive scenario along the AGB can be refined by examining stars which correspond to peculiar short-lived stages during which the star fundamentally changes ( $\mathrm{S}$ or Tc stars), or to one of the most advanced stages of evolution ( $\mathrm{OH}$ emitters).

\subsubsection{Tc stars}

According to the models, the $s$-elements processed in a star can be brought to the surface by convective dredgeups. When a sufficient quantity of $s$-elements has been brought to its surface, an O-rich star becomes an $\mathrm{S}$ star. $\mathrm{S}$ stars present a $\mathrm{C} / \mathrm{O}$ ratio close to 1 and are generally considered as a transition phase between O-rich and C-rich stars. 
Some S stars are enriched in Tc, indicating that this material was brought to the surface by recent (in the last few million years) dredge-ups, as modeled by Mowlavi (1998) in agreement with the results obtained by Van Eck et al. (1998) who compared Tc and no-Tc S stars. Our list of Tc-rich S LPVs is taken from Van Eck's thesis (1999).

On another hand some O-rich LPVs (i.e. LPVs classified with an $\mathrm{M}$ spectral type) can also be enriched in Tc. Such peculiar stars were studied by Little et al. (1987) and they offer great potential as a possible constraint on the modelization of dredge-ups. Table 1 shows the individual estimated absolute magnitudes and the assigned group of these Tc stars.

We would like to highlight the correlation between location in the plane $(12,25)$ of Tc LPVs and the limit between O-rich and C-rich regions, regardless of their S or M spectral type (see Fig. 4). The only exception is R And, which will be discussed later in this paper.

However no differences are found between Tc O-rich and Tc S LPVs. Tc O-rich LPVs have the same 12 and 25 luminosities as O-rich stars. They are probably LPVs enriched in Tc by a recent dredge-up, but not efficient enough either to make the $\mathrm{C} / \mathrm{O}$ ratio close to 1 or to drastically alter the circumstellar envelope. Tc S LPVs are mainly assigned to disk population (10/12), which is not valid for Tc O-rich LPVs. This suggests that the dredgeup is more efficient in changing the $\mathrm{C} / \mathrm{O}$ surface ratio up to 1 for more massive stars. No definitive conclusions can be reached owing to the scarcity of Tc LPVs in the sample.

It is also important to note that all these stars are more luminous than $\simeq-6.5 \mathrm{mag}$ in $K$, in agreement with Van Eck et al. (1998), the bolometric correction for this type of stars being around $3 \mathrm{mag}$. This confirms the predicted location of the first thermal pulse (Mowlavi 1998) and the quite early operative third dredge-up on the TP-AGB (Van Eck 1999).

Finally, some individual Tc stars in our sample have specific properties that require a specific discussion:

- NQ Pup and HR Peg are both assigned to the disk 1 population. Their faint $K$ and IRAS luminosities (Table 1) are questionable but they are at the limit between O-rich and C-rich areas, like the other Tc S LPVs (Fig. 4). None of these stars show signs of dust emission (Jorissen \& Knapp 1998). They are probably among the least massive stars in the disk population;

$-\chi$ Cyg, for which Jorissen \& Knapp (1998) find questionable IRAS fluxes, presents no peculiarities here. Indeed its $25-12$ color index is close to zero but our estimated IRAS luminosities indicate the presence of an envelope. It is assigned to the disk 1 population and its location in the diagrams is compatible with a star of large initial mass in transit between O-rich and C-rich phases, after a recent dredge-up;

- $\mathrm{R}$ And can at first seem enigmatic. Assigned to the old disk population, it is the most luminous star our sample in 12 and 25 bands for its $K$ luminosity and it has almost the smallest 25-12 index (see Fig. 4). Our classification is probabilistic and thus a few stars can be misclassified, but this does not seem to be the case for $\mathrm{R}$ And. Indeed, the observed 25-12 color index is -0.9 , in agreement with that estimated from the IRAS absolute magnitudes $(-0.8)$.

This star probably has a very thick envelope and in the diagrams it is close to the $\mathrm{OH}$ LPVs belonging to the old disk population (see Fig. 5). A mass close to the limit at which an O-rich LPVs can become either an $\mathrm{OH}$ emitter or a carbon star may account for its characteristics.

\subsection{2. $\mathrm{R}$ Hor and $\mathrm{Tc}$ enrichment}

R Hor was found to be Tc enriched by Little et al. (1987) but not confirmed as such by Van Eck (1999) and it is the only Tc star assigned to the extended disk population. A Bayesian classification process can lead to some misclassification, but $\mathrm{R}$ Hor is at the limit of the O-rich LPVs area, close to two C-rich LPVs (RS Lup and V CrB) (see Table 3). Thus, its Tc enrichment is questionable.

Another point is the way of enrichment in $\mathrm{Tc}$ and in $\mathrm{C}$ of such a deficient and low mass star. V CrB was reported as probably "metal poor" by Hron et al. (1998) from ISO data. This agrees with our assignation to ED (Table 3), which seems a priori doubtful for a carbon star.

If this is confirmed, it would be an interesting constraint to evolutive models.

\subsubsection{Non-Tc S-type stars}

Some of the non-Tc S stars in our sample can be extrinsic $\mathrm{S}$ stars. These stars are not enriched in s-elements by internal nucleosynthesis and dredge-ups but by mass transfer from a more evolved companion. This is probably the case of X Aqr, BD Cam, V Cnc and SX Peg, for which a duplicity flag is given in the HIPPARCOS catalog. Except for $\mathrm{X}$ Aqr, they are in Fig. 4 at the lower limit in $K$ of the old disk population with thin envelope (ODf), to which they are assigned. Moreover, the four of them have a $K$ luminosity under -6.5 mag i.e. below the threshold of thermal pulses on AGB, confirming the Van Eck's (1999) result.

There are three other non-Tc S stars in our sample. They are the least luminous in 12 and 25 bands and have the largest 25-12 index among stars with their $K$ absolute magnitudes. AD Cyg is assigned to the bright disk and it is more luminous than all $\mathrm{C}$ stars. It is a massive star and we can assume that its evolution along the AGB is very rapid. On the other hand, in the $(K, 25-12),(K$, $12)$, and $(K, 25)$ planes, R Lyn and GZ Peg are close to the line on which AD Cyg and three extrinsic S stars assigned to disk 2 population with a thin envelope (D2f) (BD Cam, V Cnc, SX Peg) are located. These locations seem to confirm the non-Tc character of these stars, which are probably extrinsic S stars. However, given the difficulties in detecting duplicity, we failed to confirm this result. 
Table 1. Individual $K, 12$ and 25 luminosities, with assigned crossing $K$ (IRAS) group of Tc O-rich and S spectral type LPVs. Variability $(\mathrm{M}=$ Mira, $\mathrm{SR}=$ semi regular, $\mathrm{L}=$ irregular $)$ types and possible specificity $(\mathrm{Tc}=$ Technetiumstar, $\mathrm{BD}=$ bright galactic disk star) are given.

\begin{tabular}{|l|rr|r|r|rrr|r|}
\hline HIP id & \multicolumn{2}{|c}{ name } & types & group & $K$ & 12 & 25 & pec \\
\hline 8 & Z & Peg & MO & ODb & -6.69 & -8.90 & -9.58 & Tc \\
1236 & S & Scl & MO & ODb & -7.11 & -9.08 & -9.63 & Tc \\
77615 & R & Ser & MO & ODb & -6.97 & -9.57 & -10.17 & Tc \\
90493 & RV & Sgr & MO & D1 & -6.92 & -8.43 & -9.06 & Tc \\
104451 & T & Cep & MO & D1 & -8.08 & -9.99 & -10.60 & Tc \\
110736 & S & Gru & MO & D2b & -7.48 & -9.98 & -10.71 & Tc \\
1901 & R & And & MS & ODb & -6.79 & -9.69 & -10.54 & Tc \\
10687 & W & And & MS & D1 & -8.83 & -11.95 & -12.65 & Tc, BD \\
34356 & R & Gem & MS & D1 & -7.69 & -9.81 & -10.36 & Tc \\
35045 & AA & Cam & LS & ODb & -6.91 & -8.19 & -8.84 & Tc \\
38502 & NQ & Pup & LS & D2f & -6.73 & -6.97 & -7.05 & Tc \\
65835 & R & Hya & MS & D2b & -8.52 & -11.04 & -11.60 & Tc \\
87850 & OP & Her & SRS & D2b & -7.30 & -8.30 & -8.65 & Tc \\
94706 & T & Sgr & MS & D2b & -7.95 & -10.30 & -10.94 & Tc \\
97629 & khi & Cyg & MS & D1 & -7.48 & -9.71 & -9.88 & Tc \\
98856 & AA & Cyg & SRS & D2b & -8.83 & -10.66 & -11.35 & Tc \\
110478 & pi.1 & Gru & SRS & D1 & -8.16 & -9.82 & -10.59 & Tc \\
113131 & HR & Peg & SRS & D1 & -6.78 & -7.39 & -7.67 & Tc \\
17296 & BD & Cam & LS & D2f & -6.01 & -6.53 & -6.63 & \\
33824 & R & Lyn & MS & ODf & -7.82 & -7.85 & -8.03 & \\
40977 & V & Cnc & MS & D2f & -6.47 & -6.85 & -6.93 & \\
101270 & AD & Cyg & LS & D1 & -9.18 & -10.49 & -10.88 & BD \\
110146 & X & Aqr & MS & ODb & -6.68 & -8.92 & -9.57 & \\
112784 & SX & Peg & MS & D2f & -6.28 & -7.00 & -7.11 & \\
114347 & GZ & Peg & SRSa & D1 & -7.63 & -8.45 & -8.61 & \\
\hline
\end{tabular}

Although the number of extrinsic S stars in our sample prevented us from reaching any definitive conclusion, these results suggest that an extrinsic $\mathrm{S}$ enrichment can accelerate the evolution along the AGB with formation of a circumstellar envelope closer to a carbon than to a silicated composition, before any enrichment by the star's own nucleosysthesis and dredge-ups.

\subsection{4. $\mathrm{OH}$ stars}

Some O-rich LPVs are maser emitters on the radio frequencies corresponding to $\mathrm{OH}$ bands, the masers being pumped by infrared photons.

These stars emit at the principal frequencies of the main transition $(1665 / 67 \mathrm{MHz})$ and some also emit at $1612 \mathrm{MHz}$. The star is classified as OHII or OHI according to a stronger or a lower emission at the secondary frequency with respect to the main band. All of them are O-rich LPVs.

A systematic research of $\mathrm{OH}$ masers for stars in the solar neighbourhood has been carried out by Sivagnanam et al. (1989, 1990), Lewis et al. (1995) and Szymczack et al. (1995).
Individual estimated absolute magnitudes and assigned groups of $\mathrm{OH}$ stars in our sample are given in Table 2. Figure 5 shows their location in the distributions of the various absolute magnitudes. The distinction between $\mathrm{OHI}$ and $\mathrm{OHII}$ is not useful for our purposes because no difference was found between them in our analysis.

As expected, no OH star was found with a thin envelope, i.e. assigned to an froup. At a given $K$, they are the brightest in the 12 and 25 bands and all (except R Leo) have a 25-12 index corresponding to a thick circumstellar envelope. Their $K$ luminosity distribution is the same as that of non-OH O-rich LPVs. However, our previous $V$ calibration (Mennessier et al. 1999) indicates the extent to which the presence of a thick envelope induces an absorption in the visible range and confirms that after a new growth of the envelope the star becomes fainter and fainter in the visible range and turns into an $\mathrm{OH}-\mathrm{IR}$ source. All these results agree with the current model of $\mathrm{OH}$ sources: a maser emission pumped by photons of an infrared thick envelope that depends on the mass of the star and the mass-loss ratio. 
Table 2. Individual $K, 12$ and 25 luminosities, with assigned crossing $K$ (IRAS) group of OH maser emitters Mira.

\begin{tabular}{|l|rr|r|r|rrr|r|}
\hline HIP id & \multicolumn{2}{|c|}{ name } & types & group & $K$ & 12 & 25 & pec \\
\hline 11350 & R & Cet & MO & ODb & -6.69 & -9.29 & -10.34 & OHI \\
19567 & W & Eri & MO & D2b & -6.26 & -9.48 & -10.21 & OHI \\
21766 & R & Cae & MO & D1 & -8.56 & -10.80 & -11.48 & OHI \\
25673 & S & Ori & MO & D2b & -7.91 & -10.20 & -10.94 & OHI \\
26675 & RU & Aur & MO & ODb & -6.90 & -10.23 & -11.09 & OHI \\
27286 & S & Col & MO & D2b & -8.04 & -10.09 & -10.87 & OHI \\
36669 & Z & Pup & MO & D1 & -8.17 & -11.05 & -11.86 & OHI \\
40534 & R & Cnc & MO & D2b & -8.61 & -10.83 & -11.55 & OHI \\
47066 & X & Hya & MO & ODb & -6.48 & -8.52 & -9.08 & OHI \\
47886 & R & LMi & MO & D1 & -8.41 & -10.73 & -11.37 & OHI \\
48036 & R & Leo & MO & D1 & -7.67 & -9.91 & -10.18 & OHI \\
58854 & R & Com & MO & D1 & -6.80 & -8.41 & -9.22 & OHI \\
67626 & RX & Cen & MO & ODb & -6.80 & -9.12 & -9.90 & OHI \\
69346 & RU & Hya & MO & ODb & -6.49 & -8.95 & -9.76 & OHII \\
69816 & U & UMi & MO & D1 & -6.98 & -8.58 & -9.18 & OHI \\
70669 & RS & Vir & MO & D1 & -7.30 & -10.27 & -11.18 & OHII \\
74350 & Y & Lib & MO & ODb & -6.15 & -8.11 & -8.94 & OHI \\
75143 & S & CrB & MO & D2b & -8.01 & -10.43 & -11.45 & OHII \\
75170 & S & Ser & MO & D1 & -6.72 & -8.76 & -9.41 & OHI \\
79233 & RU & Her & MO & D1 & -7.93 & -10.07 & -10.93 & OHI \\
80488 & U & Her & MO & D2b & -7.95 & -11.06 & -11.64 & OHII \\
91389 & X & Oph & MO & D1 & -8.16 & -11.11 & -11.69 & OHI \\
93820 & R & Aql & MO & ODb & -7.31 & -9.48 & -10.49 & OHII \\
98077 & RR & Sgr & MO & D2b & -7.64 & -10.36 & -10.97 & OHI \\
98220 & RR & Aql & MO & ODb & -6.88 & -9.72 & -10.48 & OHII \\
114114 & R & Peg & MO & D2b & -7.35 & -10.12 & -10.79 & OHI \\
\hline
\end{tabular}

Finally, the kinematic assignation of $\mathrm{OH}$ emitters also provides information about these stars. Ten stars were assigned to the disk 1 population and they can thus be considered LPVs with massive progenitors. However we found that this population group is rather attractive for C-rich stars and repulsive for O-rich stars (Paper I). These ten $\mathrm{OH}$ emitters have a $K$ luminosity brighter than about $-8 \mathrm{mag}$. They are located in the same area as the Tc S LPVs in the $(K, 25-12),(K, 12)$ and $(K, 25)$ planes. Thus such stars probably have a mass at the limit of the capability to be sufficiently enriched in carbon by successive dredge-ups. As discussed in Sect. (4.2) some of them could be Hot Bottom Burning candidates.

The other $\mathrm{OH}$ sources of the sample, assigned to the disk 2 or old disk population, are less massive.

\section{How to explain the gap for observed O-rich LPVs}

As seen in Sect. 2.1 the distribution of O-rich LPVs is clearly bimodal in the diagrams of Fig. 1, separating shell and no-shell stars. This gap may a priori be formed by several scenarios of the circumstellar shell formation, but one must not forget that the sample selection can also induce such an effect. Therefore, we must take both physics and sampling into account when examining the validity of the proposed hypotheses.

\subsection{Several hypotheses}

Our sample is composed of variable stars observed by HIPPARCOS, i.e., selected from criteria based on visual magnitude. As seen in Paper I, this selection effect is the most prominent in comparison with the other ones: availability of $K$ magnitude and IRAS detection. A priori and with regard to the selection effects, we propose three possible explanations for the striking gap observed for O-rich LPVs in Figs. 1-3:

1. During its evolution along the AGB, the star presents two stages of variability. Initially it is irregular or semiregular of type $b$, then the pulsation stops and thereafter the star becomes variable for a second time as 
Bright Disk LPV's - LM calibrations

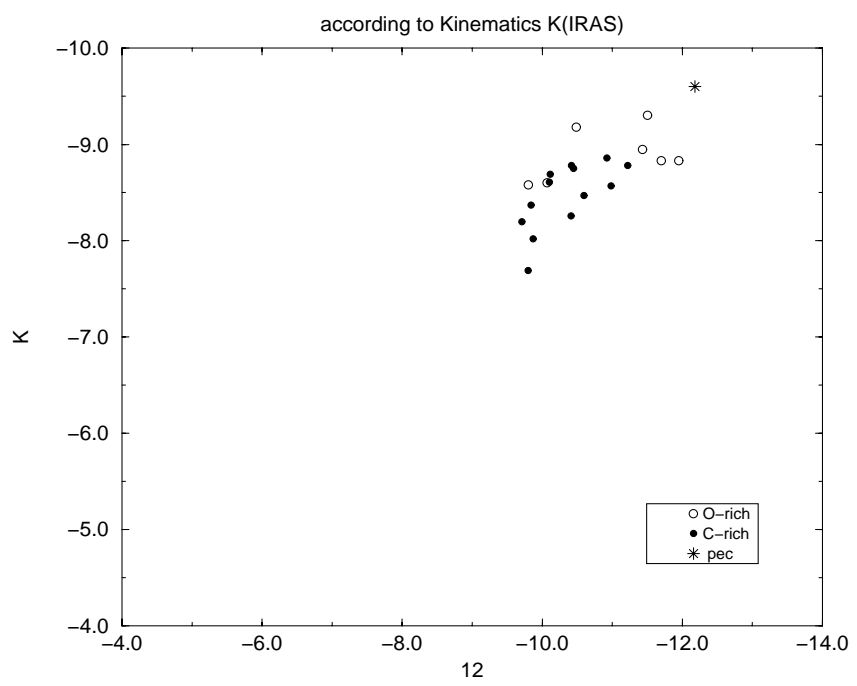

Disk1 LPV's - LM calibrations

according to Kinematics K(IRAS)

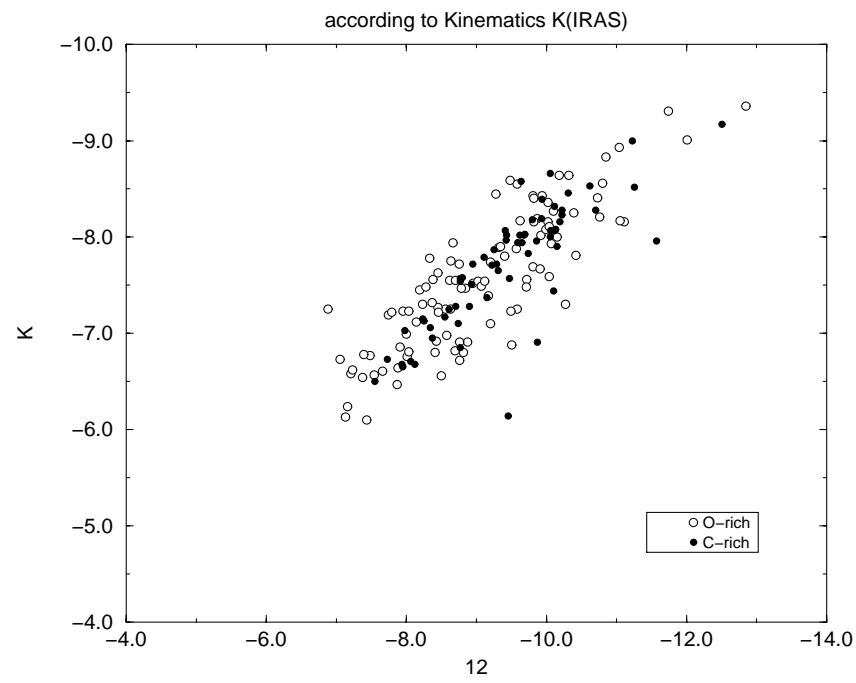

Old Disk LPV's - LM calibrations

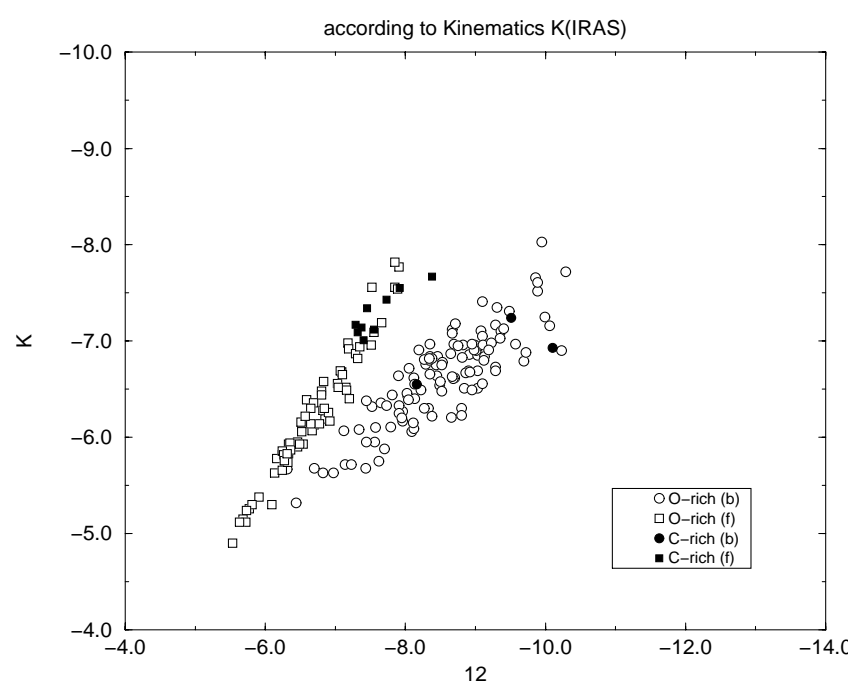

Disk2 LPV's - LM calibrations

according to Kinematics $\mathrm{K}$ (IRAS)

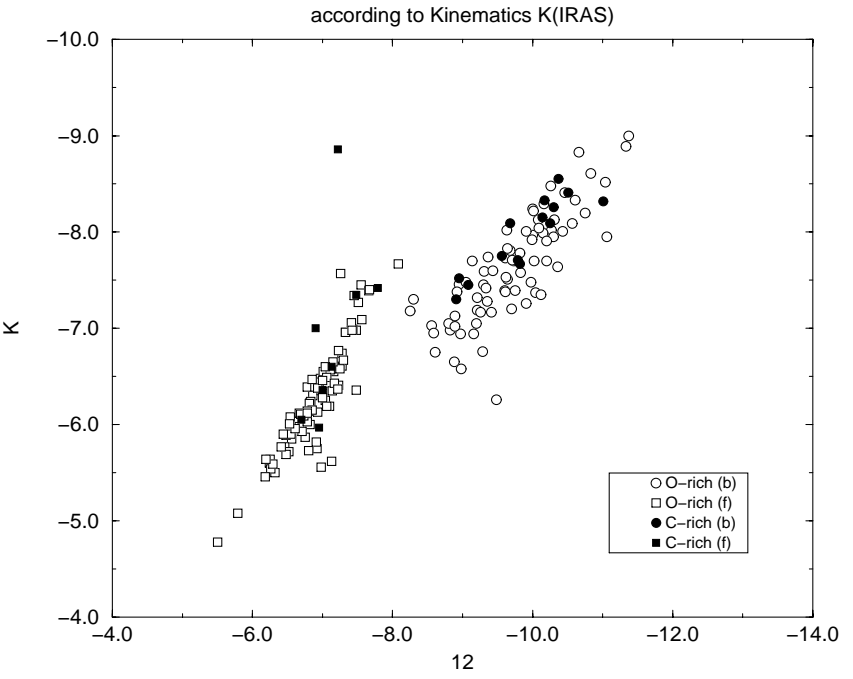

Extended Disk LPV's - LM calibrations

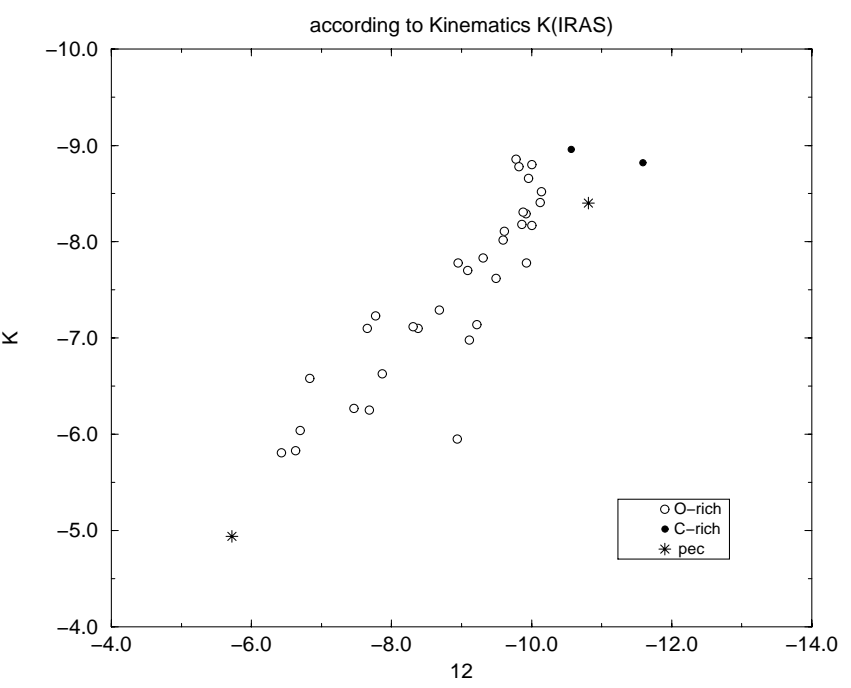

Fig. 2. Distribution of the individual estimated $K$ and 12 luminosities according to the assigned kinematical groups and to spectral types. 
Bright Disk LPV's - LM calibrations

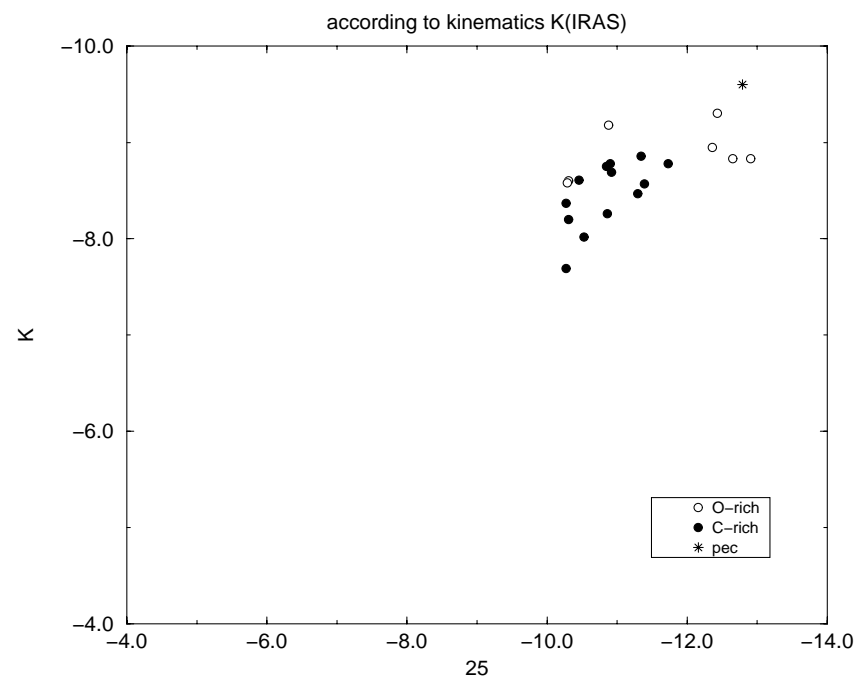

Disk1 LPV's - LM calibrations

according to Kinematics K(IRAS)

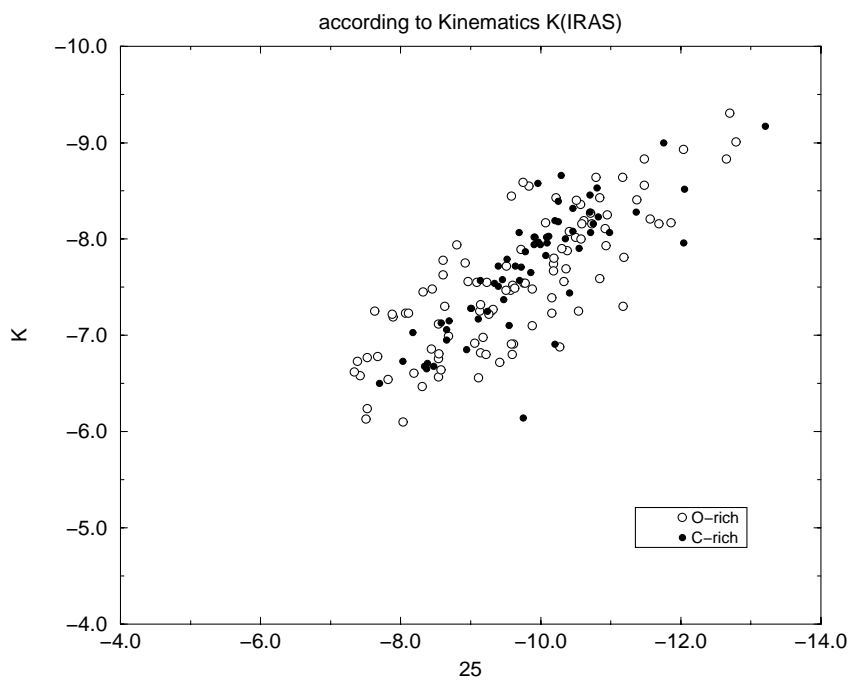

Old Disk LPV's - LM calibrations

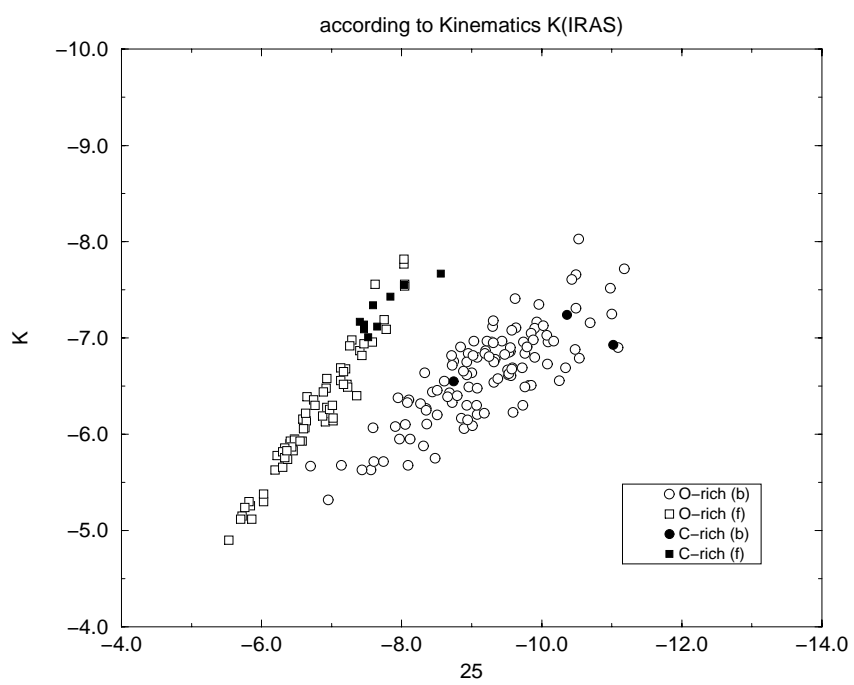

Disk2 LPV's - LM calibrations according to Kinematics K(IRAS)

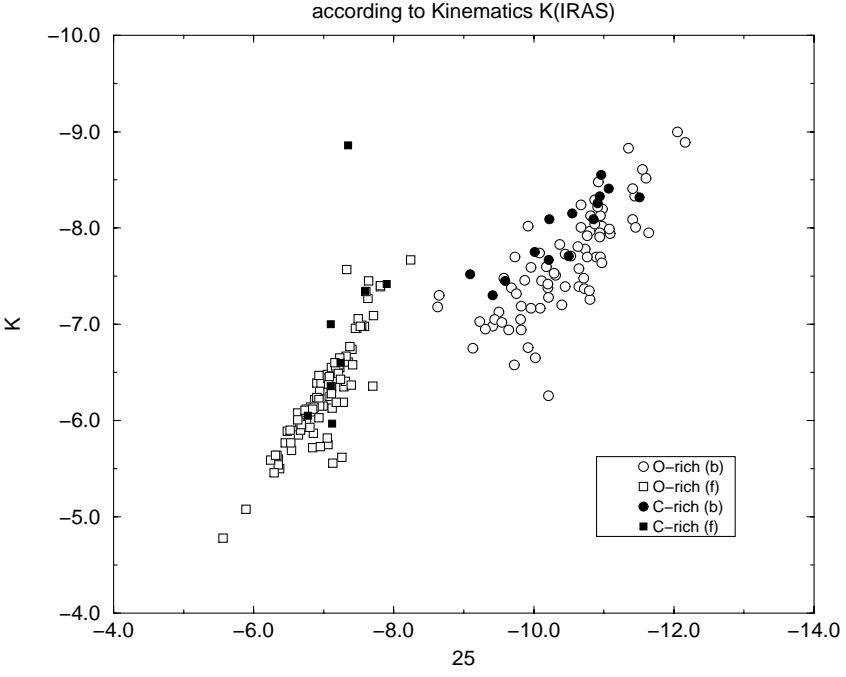

Extended Disk LPV's - LM calibrations

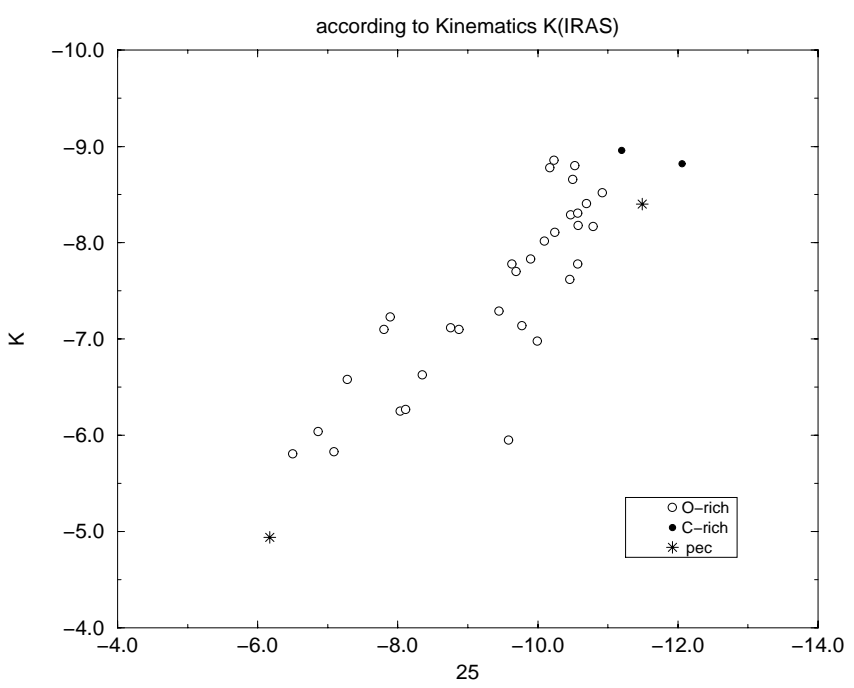

Fig. 3. Distribution of the individual estimated $K$ and 25 luminosities according to the assigned kinematical groups and to spectral types. 
a semi-regular of type a or Mira and its circumstellar envelope grows;

2. The first thermal pulses induce an irregular variability of the star. Thereafter, the pulsation becomes more regular and a significant mass loss is the source of the circumstellar envelope formation. Owing to the composition of the silicated envelope, the 12 and 25 (more 25 than 12) luminosities suddenly and strongly increase. After this rapid phase the evolution quietly continues;

3. At the beginning of the thermal pulses the SRb or irregular variables slowly develop a circumstellar envelope that grows and becomes sufficiently thick to make the star undetectable in $V$ magnitude. The envelope expands and becomes more transparent and thus the star becomes again visible in the visual magnitudes.

Obviously, reality may be more complex than these three proposed scenarios, and other explanations based on other results may be suggested. The following section is dedicated to the analysis of the three above proposed hypothesis.

\subsection{Consequences of each hypothesis}

First of all, the first hypothesis is not realistic. It has no convincing physical justification and no such behaviour is found in the models. We will thus discard it immediately.

The other two are plausible. Let us examine their coherence using Fig. 6, which shows the distributions of the individual estimated $K$ absolute magnitudes and $V-K$ indices from the $V$ and $K$ luminosities estimated for each group. Figure 6 also distinguishes the spectral types and the envelope thickness. A small number of stars assigned to the disk 1 or extended disk population have a 25-12 index close to 0 . They are marked in the Fig. 6 as "simuf" type points.

In the case of the third hypothesis, when the circumstellar envelope grows more luminous in $K$, higher values of $V-K$ are initially found; then $V-K$ decreases when the envelope expands. The star crosses back over the limit of visibility, being more luminous in $K$ and with a larger $V-K$ index.

In the case of the second hypothesis, the change in the infrared fluxes is rapid and there is no reason for a corresponding drastic change in $K$ luminosity, but the absorption in $V$ band suddenly increases. Thus, the stars belonging to the b group may be only visible (i.e. in our sample) from a more luminous lower limit in $K$ than that of the $\mathrm{f}$ group. Briefly, in the second hypothesis, from one side to the other of the gap, the star has the same $K$ luminosity but the $K$ distribution of the sample after the gap can be truncated for faint values because of an increase in $V-K$. In the third hypothesis the gap corresponds to a time for which the star is invisible. Thereafter it is brighter in $K$.

At this stage neither hypotheses 2 nor 3 can be rejected. The data available are too scarce to decide between them. The reality may be a mixture of both, but as long as these two hypotheses are considered, it is obvious that the observed gap results from a circumstellar phenomenon. On the contrary, a discontinuity in the stellar evolution along the AGB can be excluded.

\subsection{Differences according to the galactic populations}

The $(K, 12)$ and $(K, 25)$ diagrams (Figs. 2 and 3$)$ allow us to extend our analysis. They show the extent to which the evolution depends on the galactic population, i.e. on the initial mass and metallicity. We remark that the faint $K$ luminosity truncation of the sample of stars with a thick envelope assigned to disk 2 population (D2b) is far lower than the one assigned to the old disk population (ODb). This difference between disk 2 and old disk populations favours the second hypothesis. Indeed such a difference is difficult to explain by an individual increase in $K$ during the time of invisibility of the star assumed by the hypothesis 3. On the other hand, a less massive star has a less efficient mass loss with a formation of a less thick envelope and is thus less absorbed in the $V$ band. Therefore, in the case of hypothesis 2 , at a given $K$ for a thin envelope star (f), old disk population LPVs have a larger probability than disk 2 population stars to be in our $V$-selected sample just after the circumstellar envelope formation.

The location of carbon stars also reinforces hypothesis 2. Indeed, a few C-rich LPVs have a 25-12 index close to zero but they cannot be stars just reaching the AGB. This is obvious when we examine the Fig. 1 except for the 4 carbon stars belonging to the disk 2 population fainter than -7 mag. However, if we consider these 4 stars as having evolved from O-rich LPVs belonging to the truncated faint part of the $b$ distribution, everything is consistent.

\section{Peculiar evolution of bright massive LPVs}

The end of the evolution of massive LPVs on AGB induces complex phenomena and deserves a more detailed study, presented in this section.

\subsection{Luminosity boundary of $C$ stars}

The luminosity boundary of carbon stars is an important constraint for the models. We find the brightest $\mathrm{C}$ stars around $K=-9.2$, independent of metallicity (Fig. 1), in agreement with the theoretical boundary: $M_{\mathrm{bol}}=-6.4$ (Boothroyd et al. 1993).

However, in our individual estimates of absolute magnitude some O-rich LPVs are brighter in $K$ than the brightest $\mathrm{C}$ stars (see Table 3 ). This could be due to Hot Bottom Burning (HBB) that prevents carbon star formation. One of them, R Cen, is even brighter than the $3 \sigma$ upper limit of the AGB population from our calibration ( $K=-9.4$ for the disk 1 population, Paper I). The properties of this exceptional star will be a guide to investigate the massive bright LPVs. 


\section{LPV's - LM calibrations}

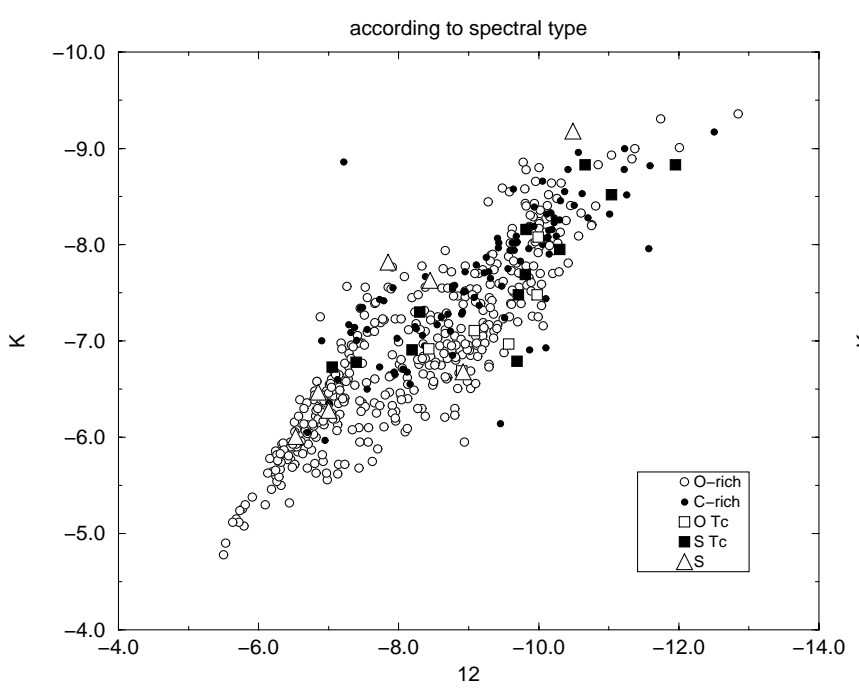

LPV'S - LM calibrations

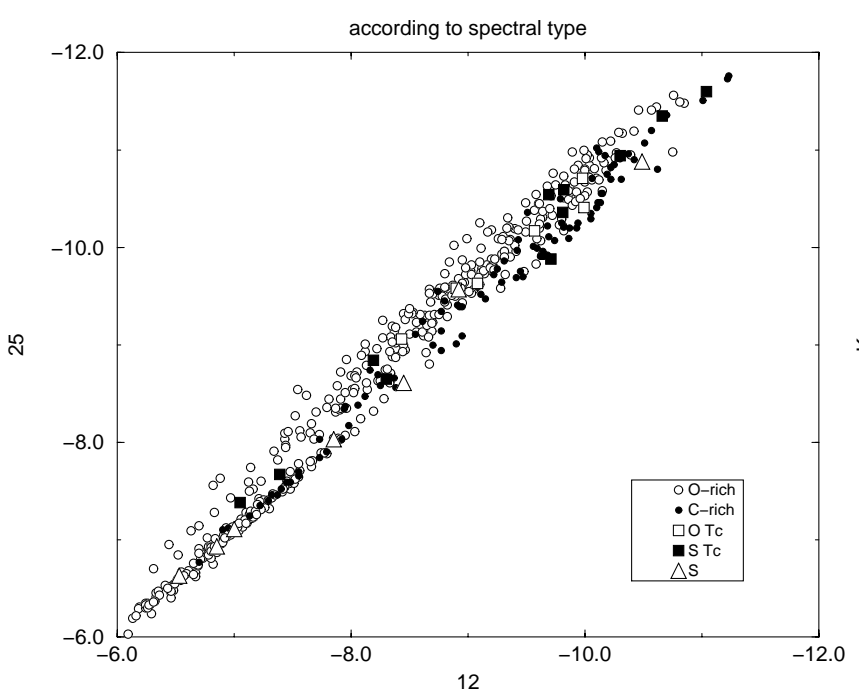

\section{LPV's - LM calibrations}

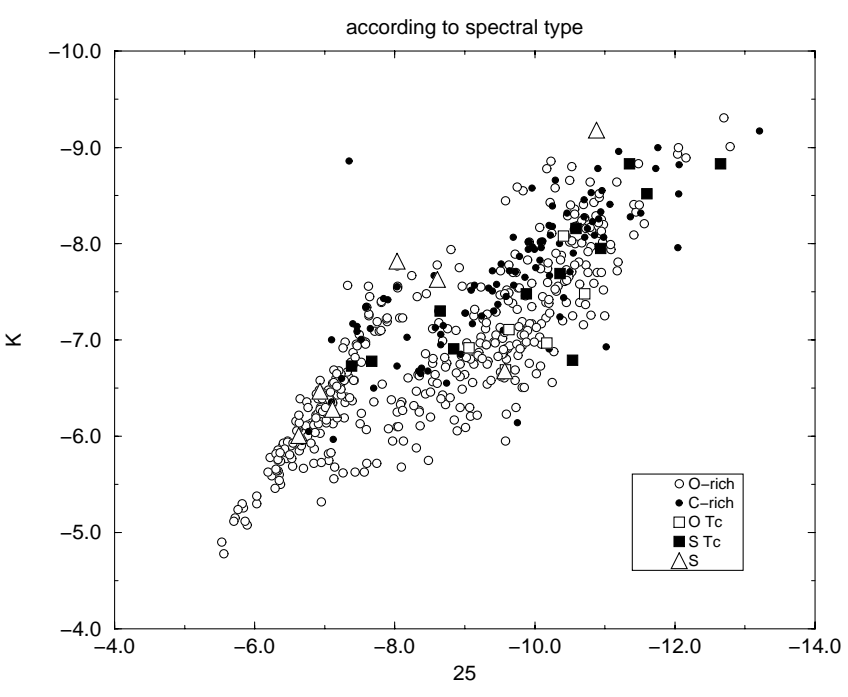

LPV's - LM calibrations

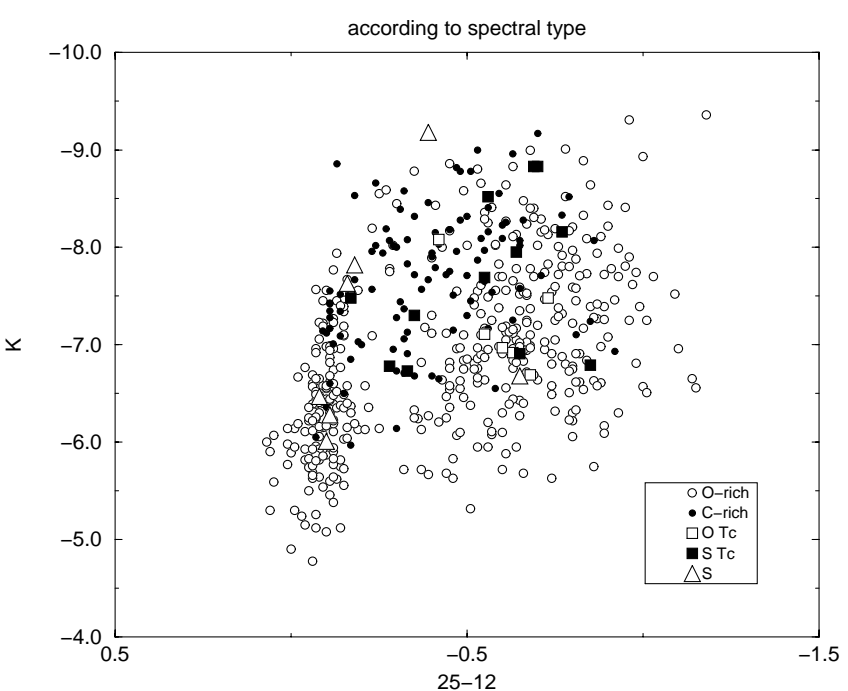

Fig. 4. Distribution of the individual estimated $K, 12$ and 25 luminosities and IRAS colors of Tc and S stars compared with O and C-rich LPVs.

\subsection{R Cen and Hot Bottom Burning}

$\mathrm{R}$ Cen has a very long period (more than 500 days) and its light curve presents a double maximum. It may have already changed from being a first overtone pulsator to a fundamental one, but this assumption does not agree with pulsation models (Ya'ari \& Tuchmann 1996) for a high luminosity star with a period around 500 days. Moreover, the hypothesis that $\mathrm{R}$ cen is a first overtone pulsator accounts for the peculiar shape of its light curve by a resonance phenomenon with the ratio $\mathrm{P} 1 / \mathrm{P} 3$ close to 2 (Barthes 1998). This author suggests that this peculiarly massive star (more than $3 \mathcal{M}_{\odot}$ and maybe $5 \mathcal{M}_{\odot}$ ) is a candidate star in the Hot Bottom Burning phase. Indeed this phenomenon stops the carbon-enrichment of the surface. This agrees with the fact that this star is nearly the brightest in $K, 12$ and 25 luminosities.

Two other candidates are proposed by Barthes (1998): $\mathrm{T}$ Cas and X Oph. In Paper I, we assign both these stars to the disk population (see Table 3), in agreement with young and initially massive LPVs. Our estimated luminosities confirm T Cas as an HBB candidate. X Oph is more intriguing because its mass is probably at the lowest limit of carbon star formation (Sect. 2.2.4).

Another peculiarity of $\mathrm{R}$ Cen is the appearance of the silicate band of its ISO-SWS spectrum (Justtanont et al. 1998). Let us examine the O-rich LPVs assigned to the disk 1 population with an estimated high luminosity. V774 Sgr and SV Cas have IRAS-LRS spectra (IRAS Science Team 1986) with a similar appearance as that of R Cen; they also have a relatively early spectral type (M5 and M6.5 respectively), and so they may also be HBB candidates. IM Cas and V539 Cas have a high luminosity, an early spectral type (M2) and their IRAS-LRS spectra are close to that of $\mathrm{R}$ Cen one in the same way as $\mathrm{T}$ Cas and X Oph. Another possible candidate could be Y Vel, which has a late spectral type LPV (M8-M9.5). 

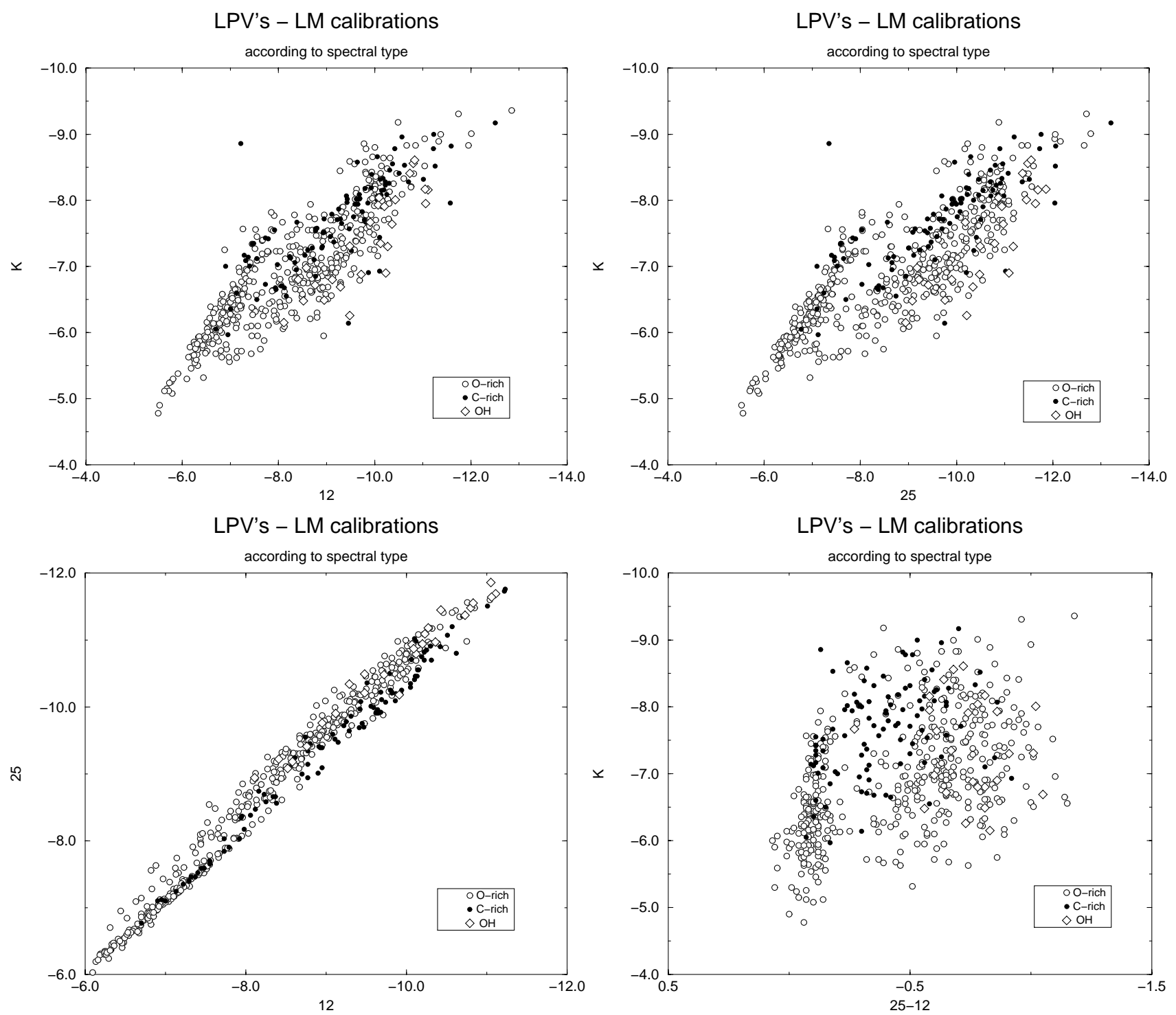

Fig. 5. Distribution of the individual estimated $K, 12$ and 25 luminosities and IRAS colors of OH stars compared to O and C-rich LPVs.

At least YZ Per has an IRAS-LRS spectrum close to that of R Cen, an early spectral type (M1-M3), but it is classified as a supergiant of class Iab and so it may be at the most advanced end of the AGB or may be a post-AGB.

IRAS luminosities and 25-12 indices of V4028 Sgr and V613 Mon indicate that they probably are in the first stages on the AGB. This is confirmed by the IRAS-LRS spectrum of V4028 Sgr in which no $\mathrm{SiO}$ feature is present. Unfortunately no IRAS-LRS spectrum of V613 Mon is available.

\subsection{R Cen and He-shell flash}

Another interesting property of $\mathrm{R}$ Cen was studied by Hawkins et al. (2001) in a recent paper. They present evidence of a steadily decreasing period of $\mathrm{R}$ Cen from 550 to 505 days during the last 50 years. They suggest that it is caused by a He-shell flash, in a similar way to R Hya, R Aql, W Dra and T UMi (Wood \& Zarro 1981).

$\mathrm{W}$ Dra and T UMi have no available HIPPARCOS data and so we could not estimate their luminosities. $\mathrm{R}$ Aql is an $\mathrm{OH}$ emitter and a remark similar to X Oph (Sect. 4.2) applies.

Wood \& Zarro (1981) deduce a value between -5.3 and $-5.5 \mathrm{mag}$. for the bolometric luminosity of R Hya, in agreement with our estimation $(K=-8.58)$. They also find that the time scale for the period change of R Hya corresponds to a longer time after the maximum luminosity of the He-shell flash than that of R Aql. This could explain the Tc enrichment of R Hya and its M6-M9S spectral type.

The period change for R Cen is steeper than for R Hya and thus Hawkins et al. (2001) give two possible explanations: either $\mathrm{R}$ Cen is in a stage right after the beginning of the flash, with a total mass less than $2-3 \mathcal{M}_{\odot}$ or it 
Disk1 LPV's - LM calibrations

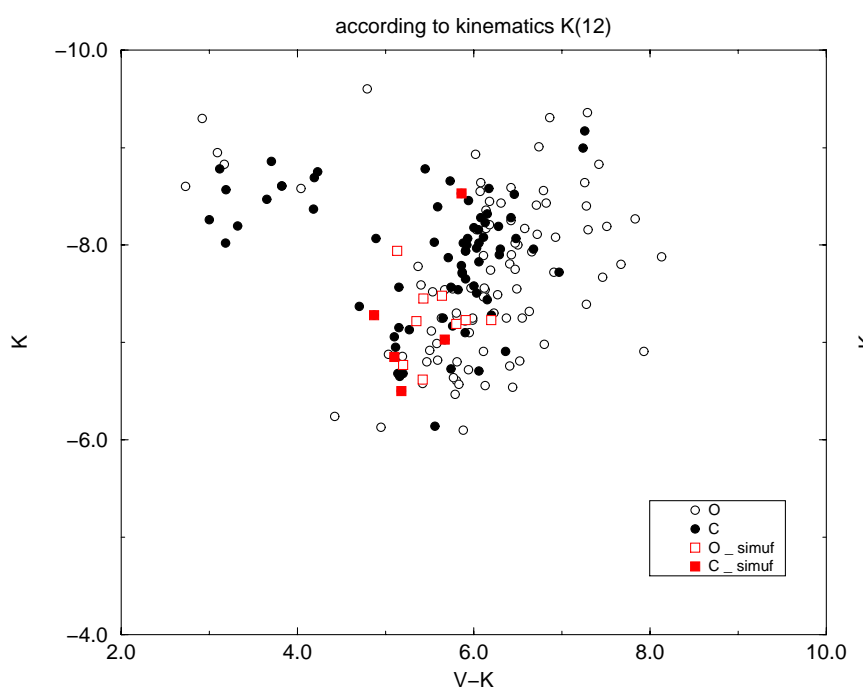

Disk2 LPV's - LM calibrations

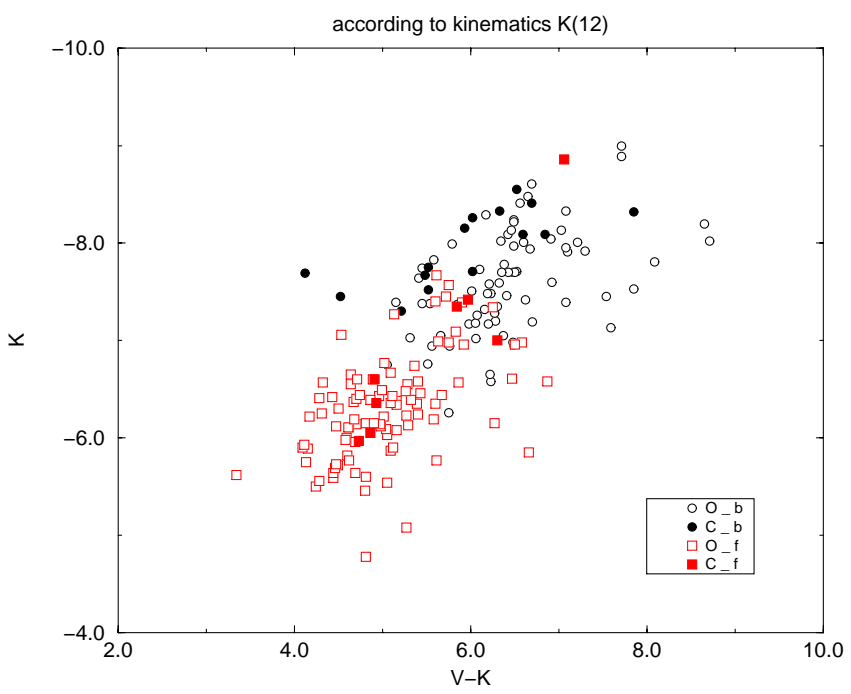

Extended Disk LPV's - LM calibrations

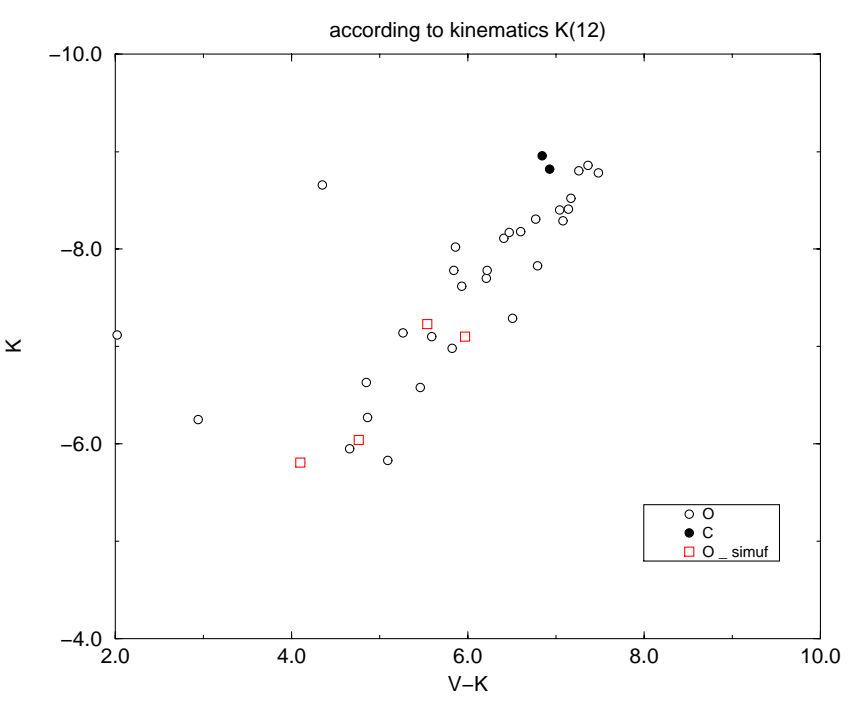

Old Disk LPV's - LM calibrations

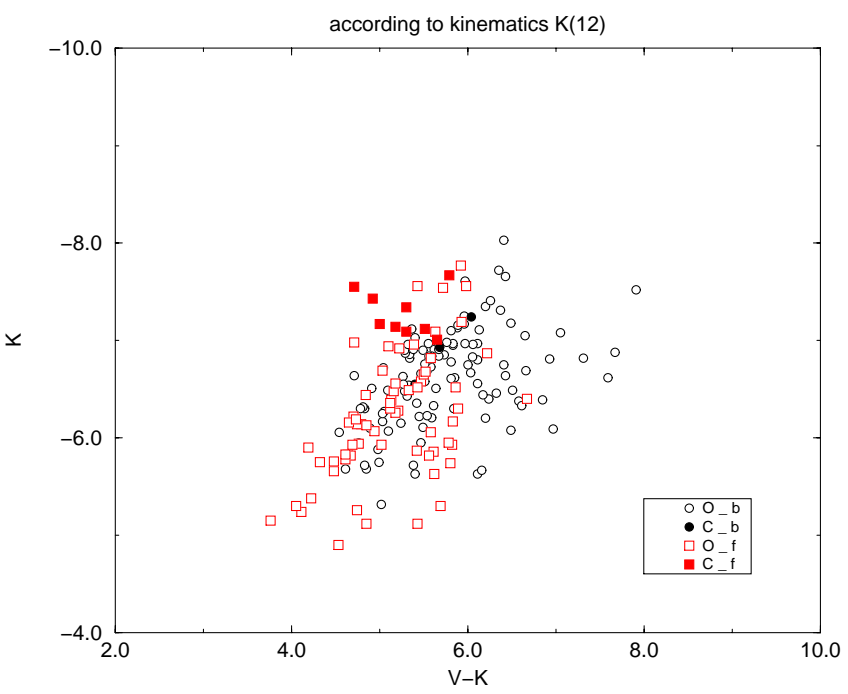

Fig. 6. Distribution of the individual estimated $K$ luminosities and $V-K$ indices according to spectral types and indication of the envelope thickness by separating stars assigned to f group or with a $25-12$ index close to 0 (simuf). Each figure corresponds to a kinematical group.

is in a stage right after where the luminosity of the flash reaches the stellar surface with a much larger range of allowed stellar mass. We find that $\mathrm{R}$ Cen is the most $\mathrm{K}$ luminous LPV but its 25-12 index is this of an S star, at the limit between $\mathrm{O}$ and $\mathrm{C}$-rich LPVs, and is assigned to $\mathrm{BD}$, the group of the most massive stars. Therefore, our results strongly favor the second possibility: $\mathrm{R}$ Cen is in a stage right after the luminosity of the flash reaches the stellar surface.

Furthermore the He-shell flash enhances the efficiency of the third dredge-up (Herwig 2000), that can explain the very peculiar location, compared to the one of that $\mathrm{HBB}$ candidates, of $\mathrm{R}$ Cen in the diagram $(K, 25-12)$. This so luminous O-rich LPV might become a carbon star exceptionally brighter than the usual luminosity limit accepted for these stars. A few such luminous carbon stars exist in the Magellanic Clouds as observed by Van Loon et al. (1999b) and modeled by Frost et al. (1998).

\section{Conclusion}

Our results confirm that the AGB evolution depends on the initial mass of the progenitor on the main sequence. The study of LPVs with peculiar properties, often associated with transition states in the stellar evolution, elucidates some points of the very complex evolution along the AGB. The simultaneous study of the behaviour of the circumstellar envelope provides further information on the evolutive state of the stars along the AGB. However, this study is mainly statistical and so results for individual stars can be erroneous because the confidence level of a probabilistic discrimination can never reach $100 \%$. 
Table 3. Individual $K, 12$ and 25 luminosities, with assigned crossing $K$ (IRAS) group, spectral $(\mathrm{O}=\mathrm{O}-\mathrm{rich}, \mathrm{C}=\mathrm{C}-\mathrm{rich}$ , $\mathrm{S}=$ Sspectral type star) and variability $(\mathrm{M}=$ Mira, $\mathrm{SR}=$ semi regular, $\mathrm{L}=$ irregular $)$ types, possible specificity $(\mathrm{Tc}=$ Technetium star, $\mathrm{OH}=\mathrm{OH}$ star, $\mathrm{BD}=$ brightgalacticdiskstar, He = star in He-shell flash) and IRAS spectrum.

\begin{tabular}{|c|c|c|c|c|c|c|c|c|c|}
\hline HIP id & \multicolumn{2}{|c|}{ name } & types & group & $K$ & 12 & 25 & pec & IRAS sp. \\
\hline 13502 & $\mathrm{R}$ & Hor & $\mathrm{MO}$ & $\mathrm{ED}$ & -8.40 & -10.81 & -11.49 & Tc? & \\
\hline 70339 & $\mathrm{RS}$ & Lup & $\mathrm{LC}$ & ED & -8.96 & -10.57 & -11.20 & & \\
\hline 77501 & $\mathrm{~V}$ & $\mathrm{CrB}$ & $\mathrm{MC}$ & $\mathrm{ED}$ & -8.82 & -11.59 & -12.06 & & \\
\hline 1834 & $\mathrm{~T}$ & Cas & MO & $\mathrm{D} 2 \mathrm{~b}$ & -9.00 & -11.37 & -12.05 & & 115 \\
\hline 7139 & IM & Cas & $\mathrm{SROb}$ & D1 & -8.95 & -11.43 & -12.36 & $\mathrm{BD}$ & 115 \\
\hline 7598 & V539 & Cas & $\mathrm{LO}$ & D1 & -9.30 & -11.50 & -12.43 & $\mathrm{BD}$ & 116 \\
\hline 12302 & $\mathrm{YZ}$ & Per & SROb & D1 & -8.83 & -11.70 & -12.91 & $\mathrm{BD}$ & 127 \\
\hline 15530 & UZ & Per & $\mathrm{SROb}$ & D1 & -8.93 & -11.04 & -12.04 & & 125 \\
\hline 46502 & $\mathrm{Y}$ & Vel & $\mathrm{MO}$ & D1 & -9.01 & -12.01 & -12.79 & & 123 \\
\hline 69754 & $\mathrm{R}$ & Cen & MO & D1 & -9.60 & -12.18 & -12.79 & $\mathrm{BD}, \mathrm{He}$ & 122 \\
\hline 87668 & V774 & Sgr & $\mathrm{LO}$ & D1 & -9.36 & -12.85 & -14.03 & & 129 \\
\hline 116705 & SV & Cas & SROa & D1 & -9.31 & -11.74 & -12.70 & & 125 \\
\hline 91389 & $\mathrm{X}$ & Oph & MO & D1 & -8.16 & -11.11 & -11.69 & $\mathrm{OH}$ & 115 \\
\hline 32627 & V613 & Mon & SROb & D1 & -8.60 & -10.07 & -10.31 & $\mathrm{BD}$ & \\
\hline 89980 & V4028 & Sgr & SROq & D1 & -8.58 & -9.80 & -10.29 & $\mathrm{BD}$ & \\
\hline 65835 & $\mathrm{R}$ & Hya & MS & $\mathrm{D} 2 \mathrm{~b}$ & -8.52 & -11.04 & -11.60 & $\mathrm{Tc}, \mathrm{He}$ & \\
\hline 93820 & $\mathrm{R}$ & Aql & MO & $\mathrm{ODb}$ & -7.31 & -9.48 & -10.49 & $\mathrm{OH}, \mathrm{He}$ & \\
\hline
\end{tabular}

The proposed evolutive scenario, schematically represented in Fig. 7, is:

- At the beginning of the variability phase, stars belonging to the disk 2 or old disk population (i.e., with a not too large initial mass $\mathcal{M}_{\mathrm{ms}}$ ) are irregular L or semi-regular SRb O-rich variables. They begin to slowly produce an envelope. Thereafter the envelope expands, and the IRAS luminosities of the star rapidly grow brighter. They become O-rich SRa or Mira. Depending on smaller or greater $\mathcal{M}_{\text {ms }}$, they can evolve either to $\mathrm{OH}$ emitters and finally $\mathrm{OH}-\mathrm{IR}$ sources or, for the more massive, to be enriched in s elements and then in carbon by successive dredge-ups and finally become a C-rich Mira. The least massive stars leave the AGB with no special transformation of their surface abundance;

- The disk 1 and bright disk population LPVs seem to very rapidly build a bright expanding envelope. They can also evolve to $\mathrm{OH}$ or C-rich stars. Although this population is not attractive for O-rich LPVs, a tenth of $\mathrm{OH}$ Mira belong to it. They are probably not massive enough to be sufficiently carbon enriched. A few may be HBB stars.

If the mass of the star is high enough, after a number of dredge-ups the external shells of the star can be enriched in carbon. The $\mathrm{C} / \mathrm{O}$ ratio becomes larger than 1. When $\mathrm{C} / \mathrm{O}$ is around 1, the star is an $\mathrm{S}$ star.
At the same time, strong changes take place in the circumstellar envelope, which becomes dominated by carbonated grains, and the 25-12 index increases in conformity with the loop drawn in the IRAS color-color diagram, as predicted by Willems \& de Jong (1988) and calculated by Chan \& Kwok (1988). Our luminosity calibrations in 12 and 25 clearly show that the star becomes fainter in both luminosities but more in 25 than in 12. The C-rich irregular and SRb stars seem to be the most evolved and massive.

The study of LPVs enriched in Tc confirms that they are at different stages along the AGB but after a recent dredge-up. It allows us to confirm the location of the first dredge-up at $M_{\mathrm{bol}}=-3.5$ and the quite early operative third dredge-up on the TP-AGB

The no-Tc S-type LPVs (except $\mathrm{R}$ And), are faint in $K, 12$ and 25, and they are confirmed as extrinsic S stars enriched not by their own nucleosynthesis but by mass exchange from a more evolved companion. The extrinsic enrichment in $s$-elements may accelerate the evolution along the AGB and lead to the formation of an envelope closer to being carbonated than silicated before any intrinsic enrichment by successive dredge-ups.

The examination of the brightest LPVs allows us to propose a list of stars with peculiar spectral, envelope and luminosity properties that may be Hot Bottom Burning candidates. The most luminous of them, R Cen, a star 


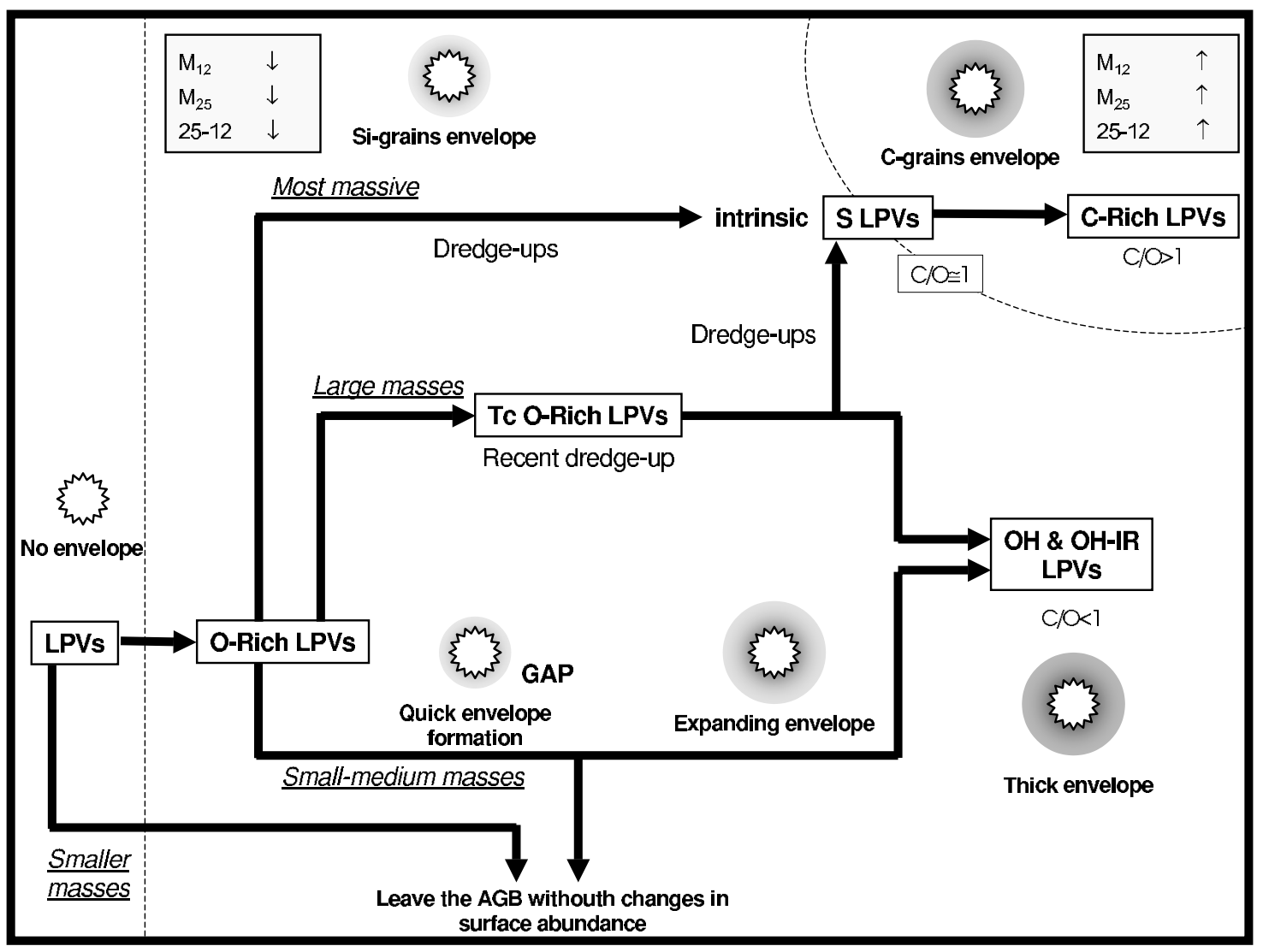

Fig. 7. Schematic illustration of the proposed stellar and circumstellar evolutive scenario.

in a He-shell flash, could become, before leaving AGB, a C-rich LPV brighter than the usual luminosity limit of carbon stars.

Acknowledgements. This study was supported by the PICASSO program PICS 348 and by the CICYT under contract ESP97-1803 and AYA2000-0937. We thank N. Mowlavi and R. Alvarez for constructive remarks and A. Gomez and S. Van Eck for fruitful discussions of our preliminary results.

\section{References}

Barthès, D. 1998, A\&A, 333, 647

Boothroyd, A. I., Sackmann, I. J., \& Ahern, S.C. 1993, ApJ, 416,762

Chan, S. J., \& Kwok, S. 1988, ApJ, 334, 362

Frost, C. A., Cannon, R. C., Lattanzio, J. C., Wood, P. R., \& Forestini, M. 1998, A\&A, 332, L17

Habing, H. J. 1989, The evolution of red giants to white dwarfs, in From Miras to Planetary Nebulae, ed. M. O. Mennessier, \& A. Omont (Éditions Frontières), 16

Hawkins, G., Mattei, J. A., \& Foster, G. 2001, PASP, 113, 501

Herwig, F. 2000, A\&A, 360, 952

Hron, J., Loidl, R., Hoefner, S., et al. 1998, Infrared spectra of C-type variables with ISO, ed. T. Le Bertre, A. Lèbre, \& C. Waelkens, IAU Symp. 191 (ASP Ed.), 181

IRAS Science Team, 1986, A\&AS, 65, 607

Jorissen, A., \& Knapp, G. R. 1998, A\&AS, 129, 363

Justtanont, K., Feuchgruber, H., de Jong, T., et al. 1998, A\&A, 330, L17

Lewis, B. M., David, P., \& Le Squeren, A. M. 1995, A\&AS, 111,237
Little, S. J., Little-Marenin, I. R., \& Bauer, W. H. 1987, AJ, 94, 981

Luri, X. 1995, Ph.D. Thesis, Univ. of Barcelona

Luri, X., Mennessier, M. O., Torra, J., \& Figueras, F. 1996, A\&AS, 117, 405

Mennessier, M. O., Alvarez, R., Luri, X., Noirhomme-Fraiture, M., \& Rouard, E. 1999, in Physics and evolution of LPVs from HIPPARCOS kinematics, AGB stars, ed. T. Le Bertre, A. Lèbre, \& C. Waelkens, IAU Symp., 191, 117 Mennessier, M. O., Mowlavi, N., Alvarez, R., \& Luri, X. 2000, A\&A, 374, 968

Mowlavi, N. 1998, in Carbon production from low- and Intermediate-Mass Stars,Abundances profiles: Diagnostic Tools for Galaxy History, ed. D. Friedli et al., PASP Conf. Ser., 147, 166

Sivagnanam, P., Le Squeren, A. M., Foy, F., \& Tran-Minh, F. 1989, A\&A, 211, 341

Sivagnanam, P., Braz, M. A., Le Squeren, A. M., \& Tran-Minh, F. 1990, A\&A, 233, 112

Szymczack, M., Le Squeren, A. M., Sivagnanam, P., Tran-Minh, F., \& Fournier, A. 1995, A\&A, 297, 494

Van Eck, S., Jorissen, A., Udry, S., Mayor, M., \& Pernier, B. 1998, A\&A, 329, 971

Van Eck, S., Binary intruders among peculiar red giant stars, Thesis, Univ. Libre de Bruxelles

Van Loon, J. T., Groenewegen, M. A., de Koter, A., et al. 1999a, A\&A, 351, 559

Van Loon, J. T., Zijlstra, A., \& Groenewegen, M. A. 1999b, A\&A, 346,805

Willems, F. J., \& de Jong, T. 1988, A\&A, 196, 173

Wood, P. R., \& Zarro, D. M. 1981, ApJ, 247, 247

Ya'ari, A., \& Tuchmann, Y. 1996, ApJ, 456, 350 